\title{
Chrysin-induced apoptosis is mediated through p38 and Bax activation in B16-F1 and A375 melanoma cells
}

\author{
ELENA PICHICHERO ${ }^{1}$, ROSELLA CICCONI $^{2}$, MAURIZIO MATTEI $^{2}$ and ANTONELLA CANINI $^{1}$ \\ ${ }^{1}$ Department of Biology, Honey Research Center, University of Rome 'Tor Vergata', Via della Ricerca \\ Scientifica 1; ${ }^{2}$ Centro di Servizi Interdipartimentale Stazione per la Tecnologia Animale - \\ STA University of Rome 'Tor Vergata', Via Montpellier 1, 1-00133 Rome, Italy
}

Received September 24, 2010; Accepted November 19, 2010

DOI: $10.3892 /$ ijo.2010.876

\begin{abstract}
Chrysin (5,7-dihydroxyflavone) is a natural and biologically active compound extracted from honey, plants and propolis. It possesses anti-inflammatory activity, antioxidant properties and promotes cell death by perturbing cell cycle progression. In this study, our attention focused on the possible role that chrysin may have as a potential anti-cancer agent, and we tested its biological activity in murine and human melanoma cell lines (B16-F1 and A375). This study demonstrated that chrysin reduced melanoma cell proliferation and induced cell differentiation in both human and murine melanoma cells through synthesis increase and intracellular accumulation of protoporphirin IX (PpIX). Furthermore, following treatments with chrysin an increase in the expression of porphobilinogen deaminase (PBG-D) was noted. This study demontrated also that chrysin induces cell death in human and murine melanoma cells through caspase-dependent mechanisms, involving down-regulation of ERK 1/2, and activation of p38 MAP kinases. Induction of cell death may be a promising therapeutic approach in cancer therapy. Our results suggest that chrysin may be considered a potential candidate for both cancer prevention and treatment.
\end{abstract}

\section{Introduction}

Flavonoids are a class of plant secondary metabolites, they are a group of more than 4,000 polyphenol compounds and possess a common phenylbenzopyrone structure $\left(\mathrm{C}_{6}-\mathrm{C}_{3}-\mathrm{C}_{6}\right)$, and are categorized into flavones, flavonols, isoflavones, flavanones and flavanols. Falvonoids are found in fruits, vegetables as well as in wine, tea and honey, and represent a common constituent of human diet (1). Flavonoids have been

Correspondence to: Professor Antonella Canini, Honey Research Center, Department of Biology University of Rome Tor Vergata, Via della Ricerca Scientifica, 1-00133 Rome, Italy

E-mail: canini@uniroma2.it

Key words: chrysin, B16-F1, A375, MAP kinases, Bax, protoporphyrine IX, SB 203580, apoptosis reported to possess many useful properties including antiinflammatory activity, anti-oxidant activity, enzyme inhibition and cytotoxic anti-tumor activity (2). Molecular studies have related that flavonoids can exert modulatory action in cell by interacting with a wide spectrum of molecular targets central to cell signaling machinery, like mitogen-activated protein kinases (MAPK) (3,4). Chrysin (5,7-dihydroxyflavone), an apigenin analog, is a naturally active compound of the flavone group extracted from honey, propolis and plants (Fig. 1). Several studies in recent years have shown that chrysin has multiple biological activities such as anti-inflammation and anti-oxidation effects $(5,6)$. It has been found that chrysin possesses cancer chemopreventive activity through arresting cell cycle progression $(7,8)$, and inducing apoptosis in different malignant cancer cells $(9,10)$.

Apoptosis (programmed cell death), is a normal component of the development and health of multicellular organism. Apoptosis also occurs as a defense mechanism such as in immune reactions or when cells are damaged by disease or noxious agents (11). Abnormalities in cell death regulation can be a significant component of disease such as cancer. Cancer is an example where the normal mechanisms of cell cycle regulation are dysfuntional, with either an overproliferation of cells and/or decreased removal of cells (12). Apoptosis is an extremely well-ordered process that is characterized by collapse of cytoskeleton, DNA fragmentation, and membrane bledding. Cells ungergoing apoptosis ultimately disassemble into membrane-enclosed vescicles (apoptotic bodies) that are engulfed by neighboring cells and phagocytes, thus preventing an inflammatory response (13). The mechanisms of apoptosis are highly complex involving a cascade of molecular events. To date, research indicates that there are two main apoptotic pathways: the extrinsic or death receptor pathway, and the intrinsic or mitochondrial pathway (14). The control and regulation of apoptotic mitochondrial events occur through the members of the Bcl-2 family of proteins (15); some of these proteins (e.g. Bcl-2 and Bcl-XL) are anti-apoptotic, while others (e.g. Bad, Bax and Bid) are pro-apoptotic. The sensitivity of cell to apoptotic stimuli can depend on the balance of pro- and anti-apoptotic Bcl-2 family proteins (16). However, the intrinsic and extrinsic pathways have in common the key molecules of apoptosis, cysteine proteases known as caspases. Caspases are divided 
into activator (caspase-2, -8, -9) and executioner (caspase-3, -6, -7) (17).

The differentiation process still represents a crucial point in development and progression of many cancer types, such as melanoma, which show also alterations in the normal cell growth program and survival time (18). In such a scenario mitogen-activated protein kinases (MAPKs, serine threonine kinases) seem to play an important role. MAPKs form a large family of serine-threonine protein kinases conserved through evolution. In mammalian cells, four distinct MAP kinase cascades have been identified: extracellular signal regulated kinases (ERK 1/2), c-Jun amino-terminal kinases (JNK), or stress-activated protein kinases (SAPK), p38 MAP kinase (p38). ERK $1 / 2$ is a member of Ras/Raf/Mek/ERK mitogenactivated protein kinase signalling pathway that regulates cell survival, proliferation and differentiation process following transmission of a wide variety of extracellular stimuli from the cell surface to the nucleus. Like ERK 1/2, p38 MAP cascade regulates a variety of cellular response associated with inflammation, cell differentiation, cell growth and death (19). The role played by different signal transduction molecules in the control of cell survival is the subject of intense discussion. The $\mathrm{p} 38$ pathways are activated in response to different apoptotic stimuli and seem to play a decisive role in this process. On the other hand the ERK cascade is one of the major kinase pathway involved in cell survival. The balance between these two MAPK pathway has been proposed to determine the cell fate (20).

Since research in melanoma cancer therapy is focused on the discovery of novel drugs able to reduce its proliferative capacity and induce terminal differentiation, in this study we have investigated the anti-proliferative activity of chrysin in murine (B16-F1) and human (A375) melanoma cell lines; moreover we identified specific markers of melanoma cell differentiation and the expression level of MAPKs (p38 and ERK 1/2) following treatment. In the present study, we investigated also if the anti-proliferative activity of chrysin is associated with apoptosis. We found that chrysin treatment promotes apoptosis through activation of caspase cascade and that p38 signalling pathway plays a crucial role in this model.

\section{Materials and methods}

Cell cultures and treatments. B16-F1 mouse melanoma cell line and A375 human melanoma cell line were purchased from American Type Culture Collection. The cells were grown in RPMI-1640 medium supplemented with $10 \%(\mathrm{v} / \mathrm{v})$ fetal bovine serum $(\mathrm{FBS}), 1 \%(\mathrm{v} / \mathrm{v}) \mathrm{L}$-glutamine, $100 \mathrm{U}$ penicillin and $100 \mu \mathrm{g} / \mathrm{ml}$ streptomycin at $37^{\circ} \mathrm{C}$ in a humidified atmosphere with $5 \% \mathrm{CO}_{2}$. The cells were passaged twice weekly. Chrysin was diluted in methanol at the final concentration of 10, 25, and $50 \mu \mathrm{M}$; the compound was added to the cells in complete medium after $24 \mathrm{~h}$ of growth. As solvent control, cells were treated with methanol. The cells were washed with PBS after different times of treatment, and harvested by centrifugation after trypsinization.

Protoporphyrine IX (PpIX) analysis of melanoma cells treated with chrysin. B16-F1 and A375 cells were seeded at a density

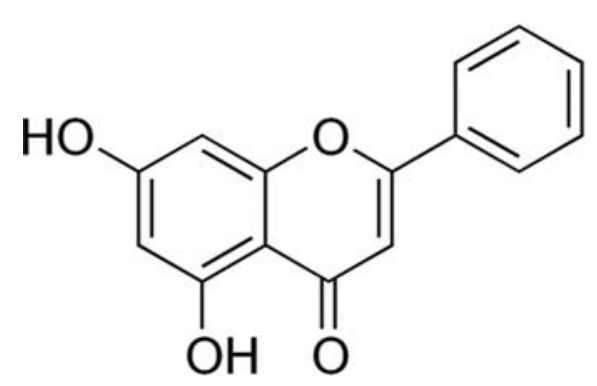

Figure 1. Chemical structure of chrysin (5,7-dihydroxyflavone).

of $1 \times 10^{4}$ cells $/ \mathrm{ml}$ in 24 -well plates. Cells were treated as previously described, after 24,48 and $72 \mathrm{~h}$ incubation cells were washed with PBS, harvested by trypsinization, centrifuged and resuspended in paraformaldehyde (PFA) $1 \%$. Samples were incubated at least $30 \mathrm{~min}$ in the dark at $4^{\circ} \mathrm{C}$, then the samples were analyzed on a FACScalibur flow cytometer using the $488 \mathrm{~nm}$ line from an argon laser (BectonDickinson, Mountain View, CA). A total of 10,000 cells were analyzed for each sample; scattering properties as well as fluorescence emitted from the cells at $670 \mathrm{~nm}$, were measured.

Caspase-3 activity colorimetric assay. B16-F1 and A375 cells were seeded at density of $2 \times 10^{4}$ cells $/ \mathrm{ml}$ in 24 -well plates. After $24 \mathrm{~h}$ cells were treated with chrysin at concentration of 10,25 and $50 \mu \mathrm{M}$. After $24 \mathrm{~h}$ of treatment and incubation at $37^{\circ} \mathrm{C}$ and $5 \% \mathrm{CO}_{2}$, cells were washed with PBS, harvested with trypsin and cetrifuged. The caspase- 3 assay was performed with a caspase-3 assay kit (Sigma-Aldrich, Milan, Italy). Lysates were incubated at $37^{\circ} \mathrm{C}$ for $2 \mathrm{~h}$. Absorbance at $405 \mathrm{~nm}$ was determined using a microtiter plate reader and the changes in caspase- 3 activity of chrysin-treated melanoma cells were determined as fold control treated cells (equals 1.0). Addition of caspase-3 inhibitor (DEVD-CHO; $50 \mathrm{nM}$ ) was used to confirm specificity.

Western blot analysis. B16-F1 and A375 cells were seeded at a density of $1 \times 10^{4}$ cell $/ \mathrm{ml}$ in 6 -well plates and treated with chrysin as previously described for $24 \mathrm{~h}$. After the treatment time, cells were washed, collected by trypsinization and used to obtain total cell lysates or purification of mitochondrial fraction. To separate the mitochondrial fraction a Mitochondrial Isolation kit (Pierce, Rockford, IL) was used. Cells were suspended in lysis buffer according to the manifacturer's instructions. To obtain total cell lysates, cells were suspendend in RIPA lysis buffer [ $1 \%$ nonidet P-40, $1 \%$ sodium deoxycholate, $0.1 \%$ SDS, $0.15 \mathrm{M} \mathrm{NaCl}, 0.01 \mathrm{M}$ sodium phosphate (pH 7.2), 2 mM EDTA, $50 \mathrm{mM}$ sodium fluoride, $0.2 \mathrm{mM}$ sodium vanadate and 100 units/ml aprotinin]. After $1.30 \mathrm{~h}$ on ice, the cell lysates were cleared by centrifugation at 13,000 $\mathrm{x} \mathrm{g}$ for $15 \mathrm{~min}$ and the resultant supernatant was used or immediately stored at $-80^{\circ} \mathrm{C}$. To estimate protein concentration of both total cell lysate and mitochondrial lysates the Bradford assay (21) was used. Forty micrograms of the protein lysates were subjected to sodium dodecyl sulphate electrophoresis on $12 \%$ polyacrylamide gels (SDS-PAGE) 
A
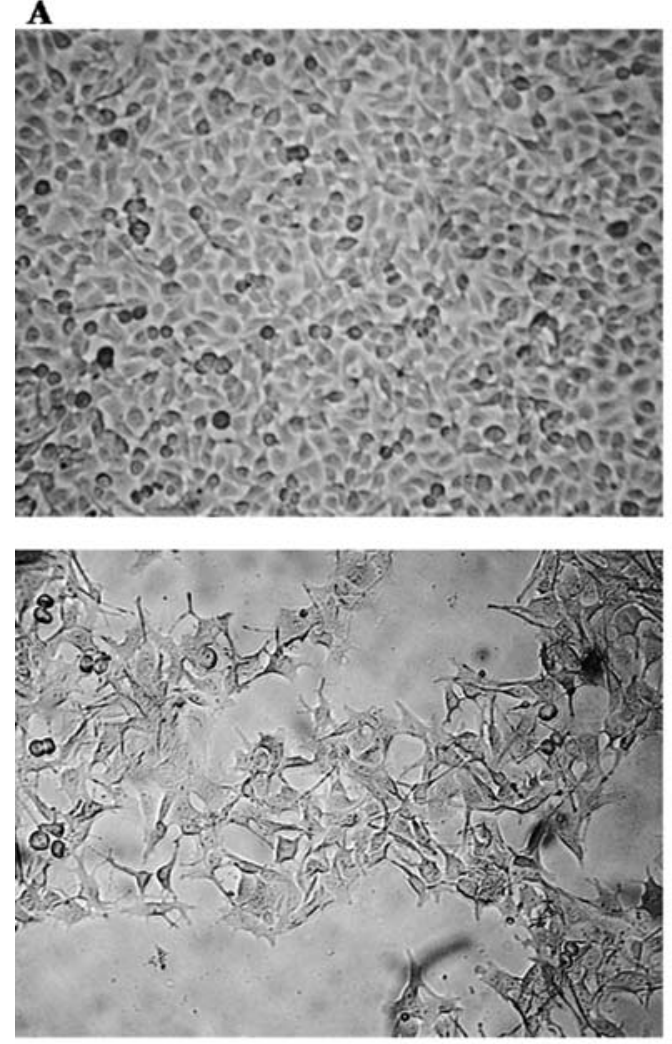

C
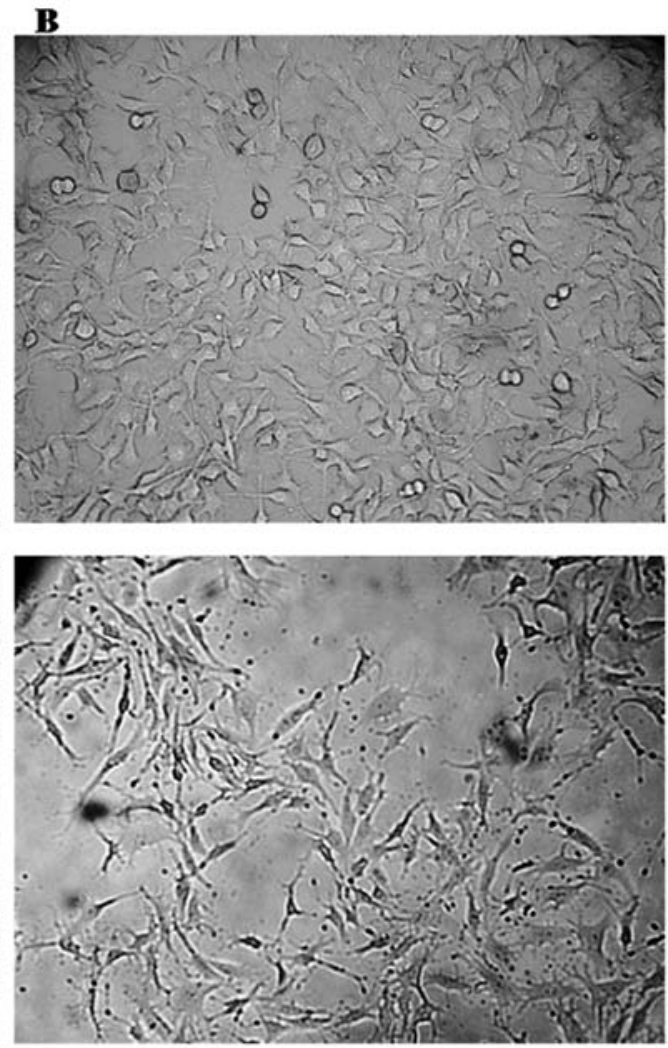

D

Figure 2. Morphological change in B16-F1 melanoma cells after 72-h chrysin treatment. (A) Untreated control; (B-D) cells incubated with 10, 25 and 50 $\mu \mathrm{M}$ chrysin. Cells were seeded and treated as described in Materials and methods, the digital images were obtained by observation with an inverted microscope at $\mathrm{x} 10$.

and the separated proteins were transferred to PVDF membrane. The blot was blocked in blocking buffer (5\% BSA/ $0.1 \%$ Tween 20 ; in TBS $\mathrm{pH} 7.5$ ) for $2 \mathrm{~h}$ at room temperature. The level of protein expression in each sample was detected using specific monoclonal and polyclonal primary antibodies diluted in $1 \% \mathrm{BSA} / 0.1 \%$ Tween 20 in TBS pH 7.5 solution overnight at $4^{\circ} \mathrm{C}$ : antibodies against phospho-p38 (Thr180/ Tyr 182), phospho-MAP kinase $1 / 2$ (pTEpY), active caspase-3 (p17 fragment) were purchased from Prodotti Gianni SpA (Milan, Italy); antibodies against PBG-D (A-16) and $\alpha$-tubulin (4G1) were purchased from Santa Cruz Biotechnology, Inc. (Santa Cruz CA, USA); antibody anti-Bax was purchased from Sigma-Aldrich (St. Louis, USA). Then the membranes were incubated with anti-mouse or anti-rabbit secondary antibodies horseradish peroxidase (HRP) conjugated and detected by chemiluminescence using the enhanced chemiluminescent substrate from Pierce (Rockford, IL) according to the manufacturer's instructions.

Trypan blue assay and mithocondrial isolation of melanoma cells treated with chrysin and p38 inhibitor SB 203580. B16-F1 and A375 cells were seeded at density of $1 \times 10^{4}$ cell $/ \mathrm{ml}$ in $24-$ well plates. After $24 \mathrm{~h}$ of growth, cells were treated with 10 , 25 and $50 \mu \mathrm{M}$ chrysin or with $10 \mu \mathrm{M}$ SB 203580 (SigmaAldrich, Milan, Italy), a specific inhibitor of p38 MAP kinase; moreover chrysin was used in combination with SB 203580. After 24, 48 and $72 \mathrm{~h}$ treatment, floating cells in the medium of each well were transferred to centrifuge tube, adherent cells were washed with PBS, collected by trypsinization and mixed with the corresponding floating cells before centrifugation. Cells were stained with $0.4 \%$ Trypan blue and counted in triplicate with an optic microscope with the aim to estimate the number of live cells. Cell survival was expressed as a percentage of treated cells with respect to appropriate vehicle-treated controls.

Melanoma cells were seeded at density of $1 \times 10^{4}$ cell $/ \mathrm{ml}$ in 6-well plates. After $24 \mathrm{~h}$ of growth, cells were treated with $50 \mu \mathrm{M}$ chrysin or with $10 \mu \mathrm{M}$ SB 203580 ; moreover chrysin was used in combination with SB 203580. After 48 h treatment, adherent cells were washed with PBS, collected by trypsinization and centrifuged. To separate the mitochondrial fraction, the Mitochondrial Isolation kit was used according to the manifacturer's instructions.

DAPI staining and localization of chrysin in melanoma cells by fluorescence microscopy. A375 and B16-F1 cells were seeded on a glass cover slip in 6-well plates and incubated with fresh RPMI-1640 supplemented with $10 \%$ fetal bovine serum for $24 \mathrm{~h}$ prior to treatment. The cells were treated with chrysin in the medium at the concetration on 10,25 and $50 \mu \mathrm{M}$. After incubation for $24 \mathrm{~h}$, cells were fixed with PFA $4 \%$ for $20 \mathrm{~min}$ in the dark at room temperature. Nuclei were counterstained with DAPI $(1 \mu \mathrm{g} / \mathrm{ml}$ in PBS $)$ and their fluorescence was monitored at $461 \mathrm{nn}$, while the autofluorescence from the 

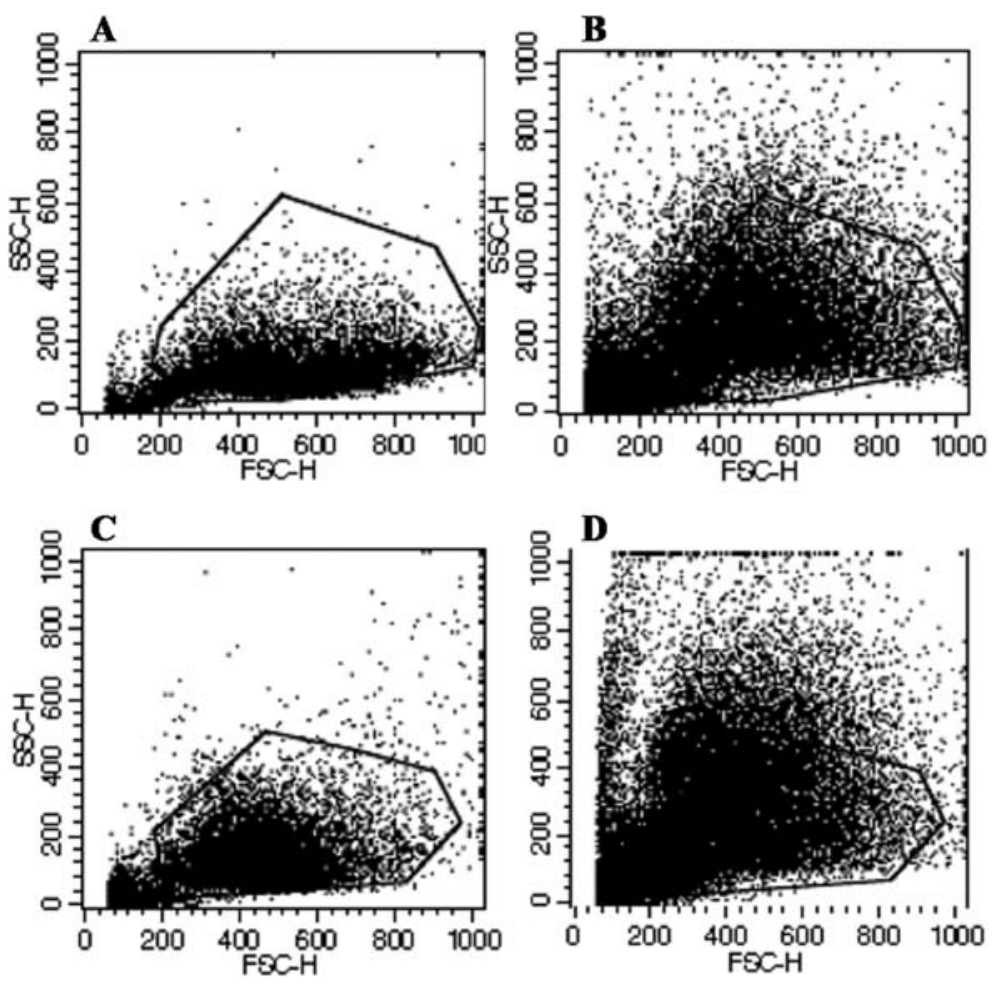

Figure 3. Light scatter of B16 (A and B) and A375 cells (C and D) incubated or not with $50 \mu \mathrm{M}$ chrysin for $72 \mathrm{~h}$. In the scattergrams chrysin-treated cancer cells (B-D) exhibited less forward scatter and much more side scatter than control cells (A-C).

flavonoid was monitored at $530 \mathrm{~nm}$, in a Leica DM-IL phase contrast microscope. The images were taken with a Leica DC-300 camera at a magnification of x100 and analyzed with Leica Image-Manager 500 software.

\section{Results}

Chrysin induces phenotypic alteration and morphological change in melanoma cells. B16-F1 cell analysis by digital images (Fig. 2) showed a dose-dependent decrease of cellular density in the culture treated with different concentrations of chrysin. Moreover, a clear morphological change of cells was detected, since the treatment induced the formation of dendritelike projections, which gave a star-like shape to the cells compared to rounded untreated cells. This effect was more evident with the increase of treatment time and concentration. Similar results were obtained for A375 cells regarding cellular density, but generally morphological changes were less evident than those obtained in B16-F1 cells (data not shown). Further phenotypic alterations were observed through cytofluorometric analysis of both treated melanoma cell lines. A change in light scatter properties of B16-F1 and A375 cells treated with chrysin was detected. In particular, a decrease of FSC (forward scatter) mean values with respect to the solvent control was observed; at the same time, SSC (side scatter) mean values increased strongly following the treatment. In Fig. 3 results relative to the treatment with a $50 \mu \mathrm{M}$ concentration for $72 \mathrm{~h}$ are shown. These effects were time- and dose-dependent (data not shown).

Chrysin treatment induces accumulation of protoporphirin IX (PpIX) and alteration in expression of porphobilinogen diaminase $(P B G-D)$ in melanoma cells. The morphological changes observed by light microscopy and the phenotypic changes highlighted by flow cytometer analysis suggested that treatments with chrysin induce differentiation in melanoma cells. To confirm this hypothesis the production of PpIX following chrysin treatments was investigated. In recent studies it has been suggested that cellular differentiation is related to an increase of intracellular PpIX; this compound is synthesized during heme biosyntesis and considered a differentiation marker for some cancer cell lines, including B16-F1 cells (22). As shown in Fig. 4, PpIX accumulation was enhanced following an increase of concentration of compound for both B16-F1 (Fig. 4A) and A375 (Fig. 4B) cell lines. Treated melanoma cells emitted a PpIX fluorescence higher than untreated control, owing to a synthesis increase and intracellular accumulation of PpIX during treatment. The increase in PpIX production and accumulation was observed in dose- and time-dependent manner. After 24, 48 and $72 \mathrm{~h}$ incubation $50 \mu \mathrm{M}$ chrysin, the increase of PpIX was 2.7-, 4.5and 6.4-fold in B16-F1 cells compared to the control. Similarly in A375 the increase of PpIX was 1.6-, 2.6- and 4.7-fold compared to control cells. To confirm fluorescence results the expression of PBG-D was investigated. PBG-D catalyse the conversion of porphobilinogen in hydroxymethylbiliane and it is one of the rate-limiting enzymes of the heme biosynthesis pathway (23). As shown in Fig. 5, Western blot analysis revealed an increase in total PBG-D protein expression in a dose-dependent manner, in both murine and human melanoma cells after $72 \mathrm{~h}$ incubation with chrysin.

Chrysin alters the expression of MAP-kinases and activates the apoptotic pathway. To explain the potential molecular 

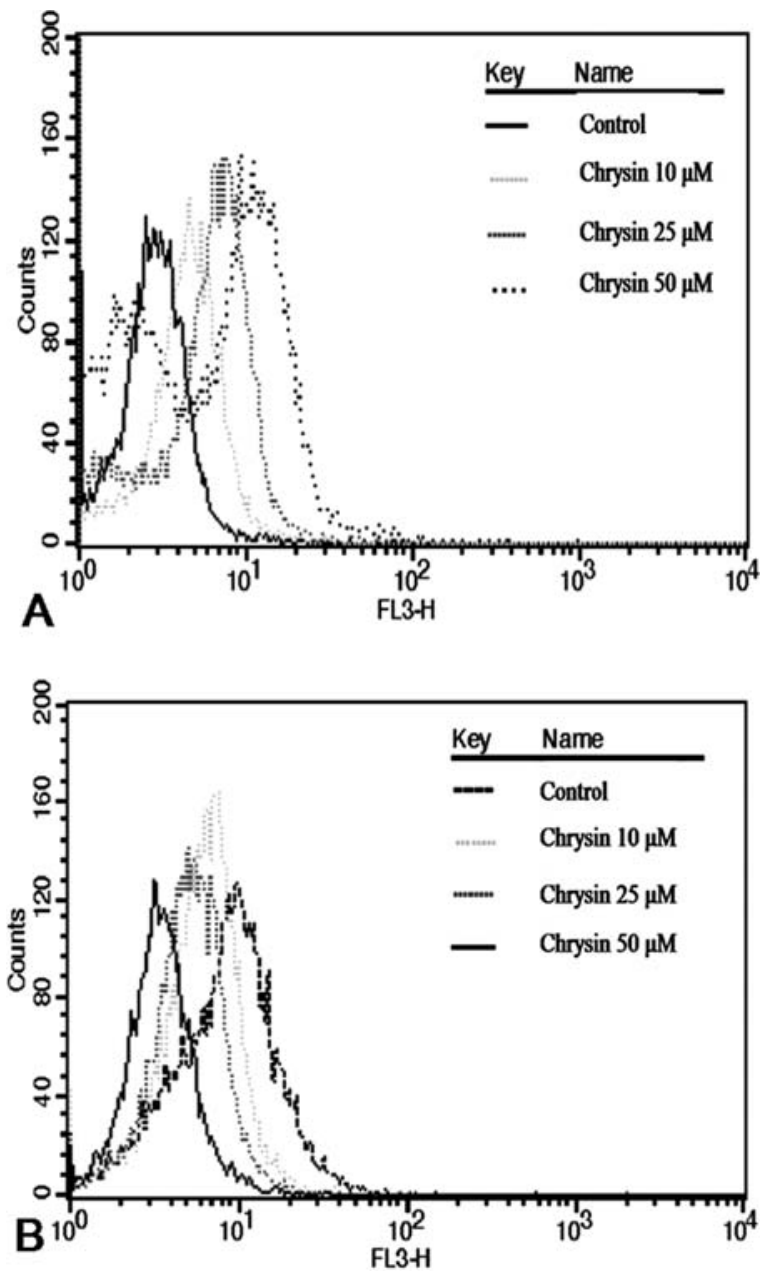

Figure 4. Protoporphirin IX (PpIX) production in B16-F1 (A) and A375 (B) cells treated or not with chrysin for $72 \mathrm{~h}$. The data shown are representative of 3 independent analysis.

mechanisms underlying the effects observed following chrysin treatment, the alterations in the state of activation of two MAPKs such as, the extracellular signal-regulated kinase (ERK1/2, p44/p42), and p38 were analyzed. Apoptosis signalling pathway was also investigated. The effects of chrysin on ERK 1/2 and p38 phosphorylation in both melanoma cell lines after $24 \mathrm{~h}$ treatment are shown in Fig. 6.

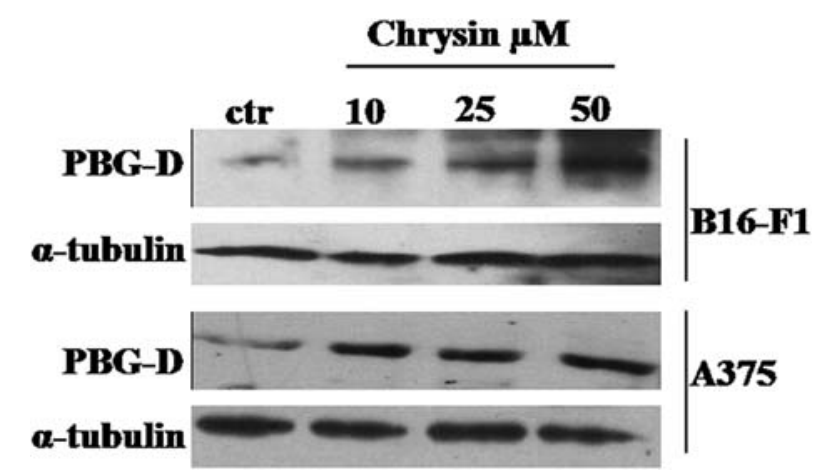

Figure 5. The effect of chrysin on PBG-D expression in B16-F1 and A375 melanoma cell lines following $72 \mathrm{~h}$ treatment. Cells were incubated with or without chrysin, after treatment, total cell lysate were prepared and subjected to SDS-PAGE followed by Western immunoblotting as described in Materials and methods. Ctr, untreated control. The data presented here are from a representative experiment repeated 3 times with similar results.

As showed, chrysin treatment altered the state of activation of ERK 1/2 in a dose-dependent manner resulting in a moderate to complete inhibition. On the other hand, chrysin treatment increased the phosphorylation state of p38 MAPK in a dosedependent manner. $\alpha$-tubulin levels were used as an internal protein control. To understand if chrysin induce programmed cell death, both the sub-cellular redistribution of Bax and the state of activation of caspase-3 were analyzed. Western blot analysis showed a different sub-cellular localization of Bax comparing chrysin treated and untreated melanoma cells (Fig. 7). After $24 \mathrm{~h}$ of treatment there is a relocation of Bax in the mitochondrial fraction. All the concentrations of chrysin increased the expression of Bax in the mitochondrial fraction compared with the control in both cell lines analyzed. As can be observed from Fig. 7, the increased expression of Bax in the mitochondrial fraction corresponded to a decrease in the cytoplasmic fraction. However, the level of expression of Bax in the total cell lysate does not vary between untreated control and the different treatments; these data showed that after treatment with chrysin there is no increased synthesis only redistribution of Bax. The state of activation of caspase- 3 was measured both with a colorimetric assay and by Western
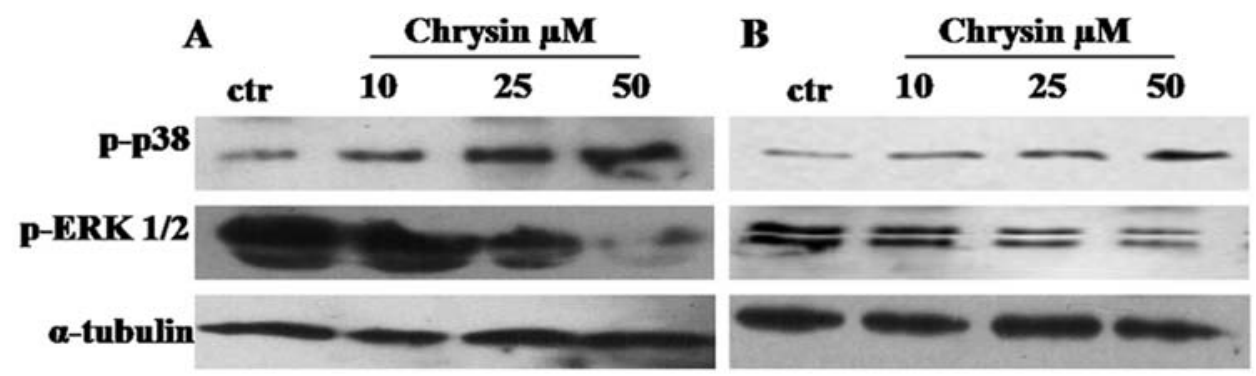

Figure 6. The effect of chrysin on ERK 1/2 and p38 activity in B16 (A) and A375 (B) cells following treatment for 24 h. Cells were incubated with or without the compound using 10,25 and $50 \mu \mathrm{M}$ doses: lanes 1 and $4(10 \mu \mathrm{M}), 2$ and $5(25 \mu \mathrm{M}), 3$ and $6(50 \mu \mathrm{M})$. After treatment, total cell lysates were prepared and subjected to SDS-PAGE followed by Western immunoblotting as described in the Materials and methods. Activation level of ERK $1 / 2$ and p38 were analyzed with their corresponding phospho-specific antibody. The data presented here are from a representative experiment repeated 3 times with similar results. 


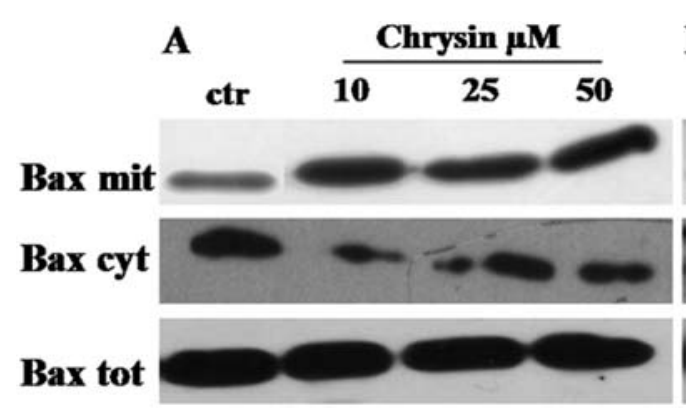

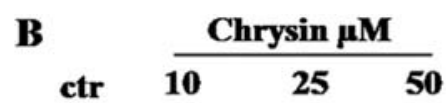

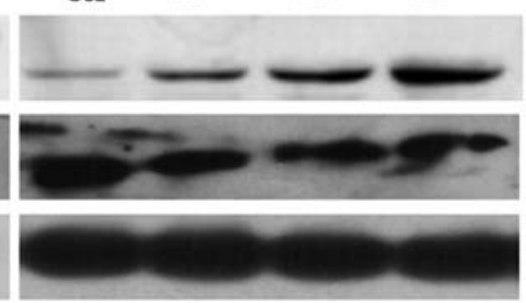

Figure 7. The effect of chrysin on Bax redistribution in B16 (A) and A375 (B) cells following treatment for $24 \mathrm{~h}$. Cells were incubated with or without the compound using 10, 25 and $50 \mu \mathrm{M}$ doses: lanes ctr untreated cells, $1(10 \mu \mathrm{M}), 2(25 \mu \mathrm{M}), 3(50 \mu \mathrm{M})$. After treatment, total and subcellular cell lysates were prepared and subjected to SDS-PAGE followed by Western immunoblotting as described in the Materials and methods. Distribution of Bax was analyzed with its corresponding antibody. The data presented here are from a representative experiment repeated 3 times with similar results.
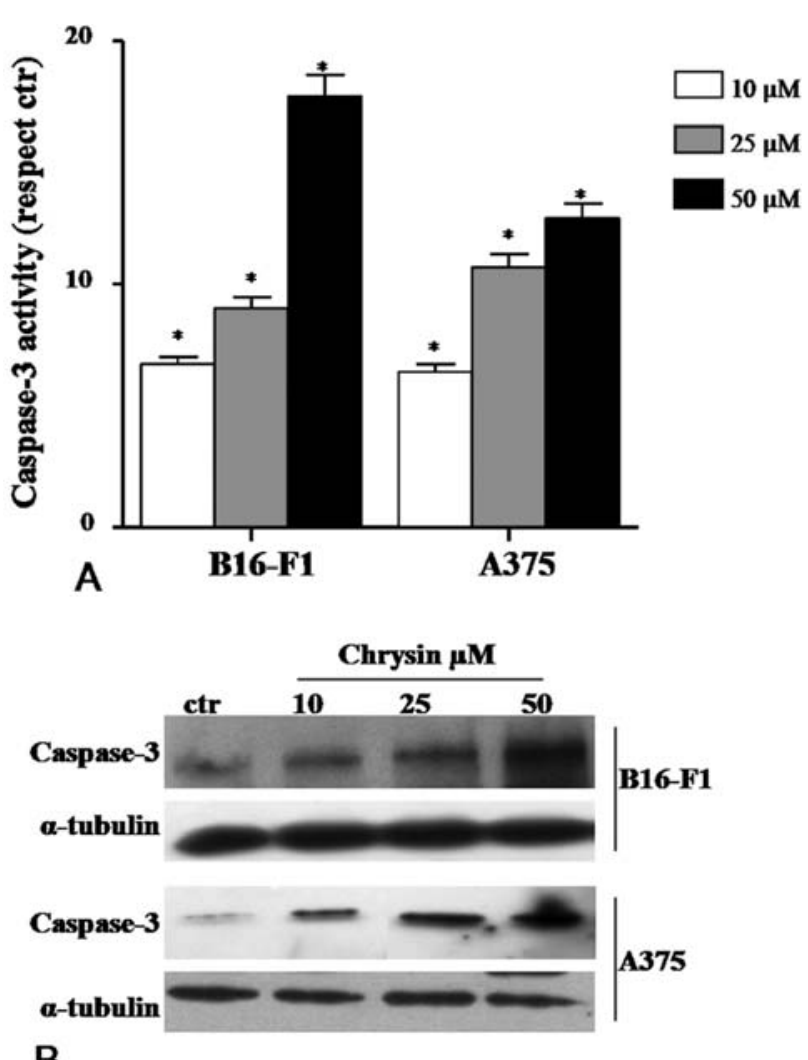

Figure 8 . The effect of chrysin on caspase-3 expression and activity in B16-F1 and A375 melanoma cell lines following $24 \mathrm{~h}$ treatment. (A) Cholorimetric caspase-3 assay; (B) Western blot with primary antibody against active caspase-3 (p17 fragment). Cells were incubated with or without chrysin, after treatment, total cell lysate were prepared and subjected to colorimetric assay or SDS-PAGE followed by Western immunoblotting as described in Materials and methods. Ctr, untreated control. (A) Results are the mean \pm SD from three indipendent experiments. ${ }^{*} \mathrm{P}<0.05$ versus vehicle control-treated cells.

blot analysis (Fig. 8). In both tests an increase in activity of this enzyme was noted in a dose-dependent manner, both in human and murine melanoma cells. The colorimetric assay of caspase activity (Fig. 8A) showed an increase of about 20 and 15 times upon treatment with $50 \mu \mathrm{M}$ chrysin in B16 and A375 cells, respectively.

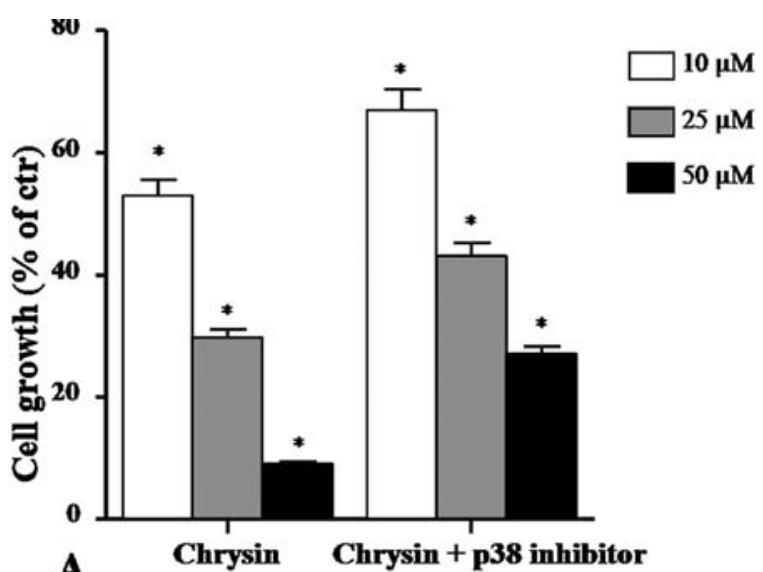

A

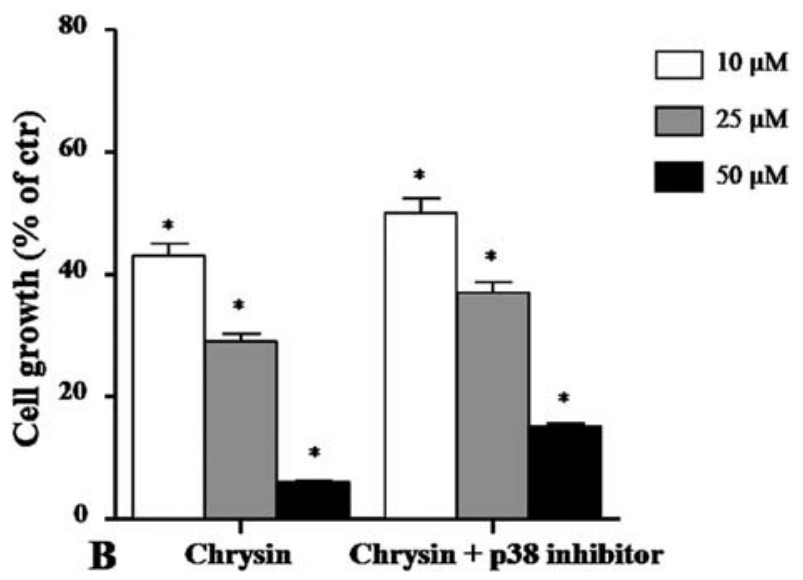

Figure 9. The effect of chrysin and p38 inhibitor (SB203580) on (A) B16-F1 and (B) A375 cell viability following $72 \mathrm{~h}$ treatment measured with Trypan blue exclusion test. Data are expressed as \% of cell survival with respect to vehicle control. Cells were incubated with chrysin at different concentrations $(10,25$ and $50 \mu \mathrm{M})$ and/or without SB203580 $10 \mu \mathrm{M}$, Trypan blue assay was carried out as described in Materials and methods. Results are the mean $\pm \mathrm{SD}$ from three indipendent experiment. ${ }^{*} \mathrm{P} \leq 0.05$ versus vehicle control.

p38 MAPK activation mediated the induction of apoptosis in melanoma cells. To investigate if p38 plays a role in the activation of apoptotic pathway in melanoma treated cells, both a Trypan blue test and Western blot analysis in presence of chrysin and SB203580 were performed. The results of 
1

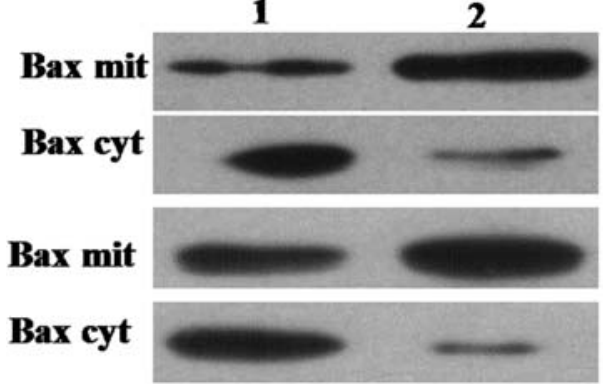

B16

$\mathbf{A 3 7 5}$

Figure 10. The effect of chrysin and p38 inhibitor (SB203580) on Bax redistribution in B16 and A375 cells following treatment for $24 \mathrm{~h}$. Cells were incubated with or without the compound using $50 \mu \mathrm{M}$ chrysin and $10 \mu \mathrm{M}$ SB203580: lanes $1(10 \mu \mathrm{M}+50 \mu \mathrm{M}), 2(50 \mu \mathrm{M})$. After treatments, total and subcellular cell lysates were prepared and subjected to SDS-PAGE followed by Western immunoblotting as described in the Materials and methods Distribution of Bax was analyzed with its corresponding antibody. The data presented here are from a representative experiment repeated 3 times with similar results.

Trypan tests showed a recovery of cell growth after cotreatment at all the treatment times (Fig. 9). This effect is most evident after $24 \mathrm{~h}$ of treatment in both cell lines. The

presence of the p38 inhibitor restores the survival rate of $73 \%$ and $78 \%$ compared with $40 \%$ growth achieved following treatment with $50 \mu \mathrm{M}$ chrysin in B16-F1 and $\mathrm{A} 375$, respectively. After $24 \mathrm{~h}$ of co-treatment a change in localization of Bax compared to the data obtained after chrysin treatment (Fig. 10) in both melanoma cell lines was also observed. The increased expression of Bax in the mitochondrial fraction after chrysin treatment was reduced when cells were treated also with p38 inhibitor (Fig. 10). Accordingly, co-treatment with $50 \mu \mathrm{M}$ chrysin and $10 \mu \mathrm{M}$ SB203580 increased levels of Bax in the cytosol.

DAPI staining confirmed the induction of apoptosis and identifies sub-cellular location of chrysin in B16-F1 and A375 melanoma cells. The images obtained by fluorescence microscopy also confirmed the hypothesis of induction of apoptosis. Apoptotic cells have condensed nuclear chromatin with or without apoptotic bodies. In Fig. 11, typical features of apoptotic nuclei can be seen, such as apoptotic bodies in the treatments with 25 and $50 \mu \mathrm{M}$ chrysin compared to control cells. These images also serve to localize chrysin in treated melanoma cells; in fact the blue fluorescence of DAPI overlapped with the green autofluorescence of chrysin because

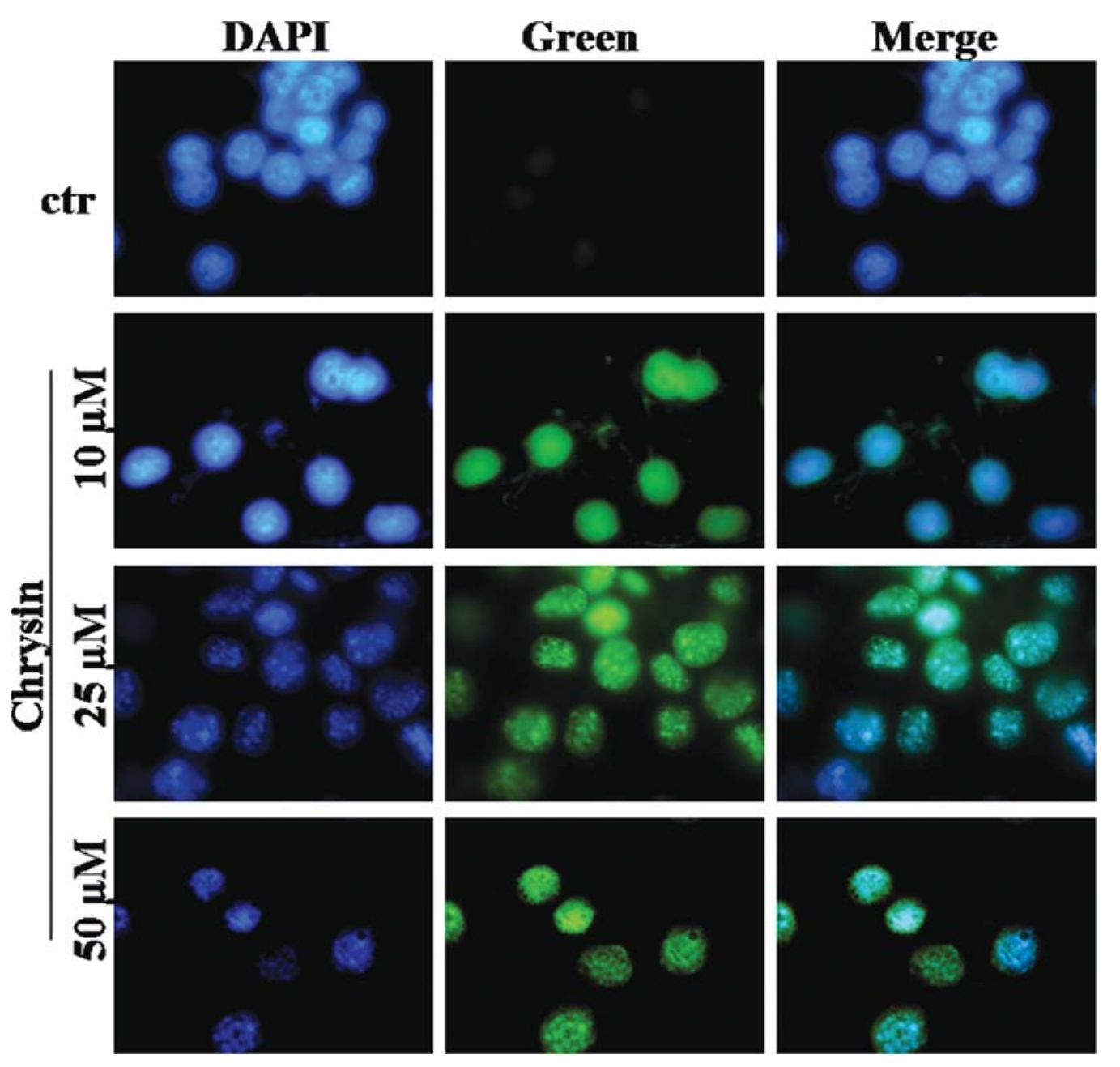

Figure 11. Subcellular localization of chrysin in B16-F1 melanoma cells. The cells were seeded on a glass cover slip in 6-well plates, treated with different concentrations of chrysin and stained with DAPI. Green fluorescence (Ex. $488 \mathrm{~nm}$ to Em. 515-535 nm) from chrysin and blue fluorescence (Ex. $358 \mathrm{~nm}$ to Em. $461 \mathrm{~nm}$ ) from DAPI were monitored under a fluorescence microscope, and the images were merged. 
cyan blue fluorescence was observed in merged images. Therefore chrysin was incorporated into melanoma cells, and accumulated in the nucleus. It can not be defined from the data whether the transportation of chrysin is conveyed through the cells in the nucleus.

\section{Discussion}

Epidemiological studies have provided data indicating that high dietary intake of flavonoids could be associated with a low cancer prelevance in human. This is supported by multitude of in vitro and in vivo studies, which show that flavonoids may inhibit various stages in carcinogenesis, namely tumor initiation, promotion and progression through many mechanisms (1). The ways in which flavonoids may act as chemopreventive agents can be divided into three distinct mechanisms: i) prevention of carcinogen metabolic activation, ii) prevention of tumour cell proliferation by inactivation or down-regulation of pro-oxidant enzymes or signal transduction enzymes, iii) by inducing tumour cell death (apoptosis) (24). The uptake and the level of metabolism of flavonoids in cancer cells played a central role in the mechanism of action of specific flavonoids. It is clear that uptake of flavonoids into cells is dependent on both the flavonoid, and perhaps more importantly, the cell type (25).

In the present study, it was demonstrated that chrysin specifically accumulated into the nucleus of melanoma cells, but the data obtained do not allow to define if the transportation of chrysin is conveyed through the cells into the nucleus. A recent study demonstated that two flavonol aglycones, such as kaempferol and ginestein, accumulated into the nucleus of Hepa-1c1cf cells (26). Previously Walgren et al (27) have been observed that flavonol aglycones easly cross cellular and nuclear membrane through passive diffusion but not endocytosis. Moreover, several studies showed that some flavonoids are able to bind aryl hydrocarbon receptor (AhR), which is a cytosolic protein. The activated AhR then translocates to the nucleus where it heterodimerizes with a protein partner (28). Different studies demonstrated that aryl hydrocarbon also can activate different MAP kinase cascades, such as p38, JNK and ERK 1/2. Generally, substrates for AhR are planar aromatic compounds with few bulky substituent groups. Flavonoids have a chemical structure similar to the planar substrates for AhR, this similarity allow flavonoids to bind to AhR and induce its activity. This activity might partially explain the potential chemoprotective effects of flavonoids (29).

In a previous study we have demonstrated that chrysin have anti-proliferative effects on human and murine melanoma cells in a dose- and time-dependent manner (8); moreover we demonstrated that chrysin induces a block of cell cycle in $G_{0} / G_{1}$ phase and an increment of cells population in Sub- $\mathrm{G}_{0}$ phase. In the present study we investigated the molecular pattern activated after chrysin treatments. The results indicated that chrysin induced cell differentiation in human and murine melanoma cells. Morphological changes were more evident in B16-F1 than in A375 cells, in fact, following incubation, B16-F1 cells showed a flattened appearence with a spread-out darken cytoplasm and extended dendrites, while the control cells appeared with a spindle-like or spherical shape form. Morphological alterations were also revealed through flow cytometry analysis. Treated melanoma cells exhibited subtle reduction of FSC compared to the control, indicating that the incident light was absorbed rather than transmitted through the cells; this is presumably due to cell size reduction or to the presence of other cellular components that absorb incident light, such as apoptotic bodies or an increased number of melanosomes. On the contrary, a high increase of SSC, indicating a raised granularity in melanoma treated cells, was observed. It is well known that some melanoma differentiation agents, such as 5,7-dime-thoxycoumarin (30), stimulate dendricity and melanogenesis after blocking cells in the $G_{0} / G_{1}$ phase similarly to tested compound. A less significant change in cellular shape was observed also in the treated A375 cell line. As a further marker of differentiation, we monitored the production of PpIX. PpIX is a photosensitiser compound synthesized and localized in the mitochondria as intermediate molecules in the heme biosynthesis pathway, of which 5-aminolevulinic acid (ALA) is the first intermediate (31). The treated melanoma cells emitted a PpIX fluorescence higher than untreated cells probably due to a synthesis increase and intracellular accumulation of PpIX during chrysin treatment. Cancer cells in vitro and in solid tumours have been reported to exhibit an enhanced capacity for PpIX syntesis facilitated by the administration of the prodrug-5-aminolevulinic acid (22). It has been reported that during the process of differentiation there is an increased accumulation of PpIX in different cell lines, such as prostate cancer cells (32), melanoma cells (33) and keratinocytes (34). It is known that most types of malignant cells accumulate more ALA-induced PpIX than the normal cells from which they arose and that changes in differentiation state are celltype specific. Furthermore, the relationship between tumour cell differentiation and photo-sensitizer accumulation is poorly defined (35). Some differentiation agents, such as butyrate, did not enhance PpIX accumulation whereas others, such as hexamethylene bisacetamide (HMBA) and methotrexate, did $(22,36)$. Following the treatment of melanoma cells with chrysin an increase in the expression of PBG-D was also noted. PBG-D is a key enzyme in heme biosyntesis and it is expressed as two isoforms, a housekeeping isoform present in all cell types and a second isoform expressed only in erythroid cells (37). Greenbaum et al (38) showed that overexpression of the human housekeeping PGB-D has been found to induce C6 glioma cell differentiation toward astrocytes. Ickowicz Schwartz et al (22) demonstrated that stimulation with HMBA induced melanocytic differentiation accompained by a prominent increase in total PBGD as well as an increase of PpIX. Systemically applied porphirin derivates are currently used for the treatment of tumours by photodynamic therapy (PDT). A new method involves the use of endogenous protoporphirin IX induced by 5-ALA. PDT is now a well-established treatment for both neoplastic and non-neoplastic disease that is based on the administration of a light-activated drug (sensitizer) followed by laser illumination of pathological areas (39). The tumor-selective effects of the PDT are based on the preferential uptake, accumulation and retention of the photosensitizer in the tumor, thus PDT induces the destruction of the tumor without causing longterm damage to normal cells (40). A combination therapy 
using a differentiation agent that specifically enhances PpIX production in cancer cells and PpIX precursor could be very useful, because tumour eradication with ALA-PDT is not always obtained. Based on this premise, our data are particularly interesting, although more studies are needed to establish the interaction between chrysin and ALA-PDT.

With the aim to elucidate the mechanism by which chrysin reduced proliferation and induced differentiation in treated melanoma cells, we focused our attention on the phosphorylation status of ERK $1 / 2$ and p38 following treatment. Using Western blot analysis, we detected a reduction in the phosphorylation of ERK $1 / 2$, following incubation of melanoma cells with chrysin; the inhibition of the MAPK activation was dose-dependent and these data were in accordance with Trypan blue assay results, since reduction of ERK phosphorylation by the treatment caused growth reduction and differentiation. It has been reported that Ras/Raf/MEK/ ERK pathway is constitutively activated, following mutation in the catalytic domain of these protein kinases, in a high percentage of human melanoma cells; particularly, an activating mutation of B-Raf that induces a constitutive phosphorylation of ERK has been detected in $66 \%$ of human melanomas (41). It is known that the treatment of melanoma cells with either U0126 (MEK 1 inhibitor) or PD098059 (MEK 2 inhibitor) causes an inhibition of cell growth and a reduction in levels of matrix metalloproteinase MMP-1 and MMP-2, involved in tumor cell invasion, demonstrating that activation of ERK is an important event in melanoma development (41). Based on this assumption, the MAPKs of Ras/Raf/ MEK/ERK signalling pathway are considered key molecular targets of novel therapy in melanoma cancer research and several agents that target this pathway are already undergoing clinical testing (42). Many studies using pharmacological inhibitors of MEK have indicated that ERK can modulate mitochondrial function, particularly those associated with cell death. For example, ERK signaling appears to promote mitochondrial ATP syntase function in glucose-deprived astrocytes, to maintain mitochondrial membrane potential and prevent cytochrome c release (19) and to inactivate the proapoptotic protein Bad (43). p38 MARK group in mammals is represented by four isoforms (p38 $\alpha, \mathrm{p} 38 \beta, \mathrm{p} 38 \gamma$ and $\mathrm{p} 388$ ) with overlapping but also distinct physiological roles (44). Activated p38 MAP kinase regulates the activity of a wide range of protein kinases (MAPKAPK2, MNK1, PRAK, MSK1), transcription factors (CHOP, p53, ATF-1/2/6, Sap1, MEF2, ELK1 and others) and some other proteins, which then further regulate the activity of their targets. This complicated network of interacting proteins is responsible for different cell activities, such as apoptosis, cell cycle arrest, cytokine production, cell differentiation, cell senescence and tumour supression (45). p38 $\alpha$ and/or p38ß were found to play an important role in cell differentiation for several different cell types. The transcription factors C/EBP, CREB, and $\mathrm{MEF} 2 \mathrm{C}$ were suggested to be downstream of the p38 pathway and would participate in the process of differentiation (46). Using Western blot analysis, we detected an increment in the phosphorylation of p38; the activation of the p38 MAPK was dose-dependent and the data were in accordance with Trypan blue assay results. There are some evidence for pro-apoptotic and anti-apoptotic role of p38 MAPKs, depending on the cell type and the stimuli $(47,48)$. The ways by which p38 contributes to an enhanced pro-apoptotic response include different mechanisms such as the phosphorylation and translocation from the cytosol to mitochondria of proteins from the Bcl-2 family, such as Bax, which leads to the release of cytochrome c from the mitochondria (49). Western blot analysis showed that following chrysin treatment the activated Bax became concentrated within the mitochondrial fraction and was reduced in the cytosolic one. To explore a potential link between p38 phosphorylation and Bax activation, melanoma cells were pretreated with a selective inhibitor of p38 (SB203580) prior to chrysin treatment. The results revealed that when $\mathrm{p} 38$ phosphorylation was pharmacologically inhibited, chrysin failed to initiate a significant change in Bax activation and translocation. The mechanism by which p38 $\alpha$ induces Bax activation is presently unknown. Most recently, Bax activation has been demonstrated in hepatoma cell lines via p38dependent phosphorylation, however, it is still unclear whether p38 and Bax can directly interact with each other (50). Bax translocation to the mitochondria has been shown to reduce mitochondrial membrane potential, enhance cytochrome c release from the mitochondria, thus activating the caspase cascade (51). The results obtained from both the Western blot and the colorimetric assays showed an increase in the activity of caspase-3, confirming that activation of $\mathrm{p} 38$, after chrysin treatment, modulates the cellular localization of Bax and the subsequent activation of programmed cell death. In viable cells a substantial portion of Bax is monomeric and found either in the cytosol or loosely attached to membranes. Following a death stimulus, cytosolic and monomeric Bax translocates to the mitochondria where it becomes an integral membrane protein and cross-linkable as a homodimer (52). Caspase- 3 is a key factor in apoptosis execution, once activated this protein breakdown or cleave key cellular components that are required for normal cellular function including structural proteins in the cytoskeleton and nuclear proteins such as DNA repair enzymes. The caspases can also activate other degradative enzymes such as DNases, which begin to cleave the DNA in the nucleus (53). The induction of apoptosis after chrysin treatment was confirmed also by DAPI staining and fluorescence microscopy analysis. Melanoma cells treated with chrysin showed the typical feature of apoptotic nuclei with the presence of apoptotic bodies in treated-cells compared to control.

In conclusion, the present study demonstrated that chrysin induces cell death in human and murine melanoma cells through caspase-dependent mechanisms, involving downregulation of ERK 1/2, and activation of p38 MAPK. Activation of the apoptotic pathway has been related to a rapid p38 MAPK activation and intracellular redistribution of pro-apoptotic Bax protein to mitochondria. Induction of cell death may be a promising therapeutic approach in cancer therapy. Although flavonoids have been studied for about 50 years, the cellular mechanisms involved in their biological activity are still largely unknown. The modulation of drugmetabolizing enzymes by flavonoids is important in terms of human health, since these enzymes can inactivate carcinogens, which contributes to cancer preventive properties of these compounds. Additionally, flavonoids may also interact whith chemotherapeutic drugs used in cancer treatment through 
the induction or inhibition of their metabolism. Our results suggest that chrysin may be considered a potential candidate for both cancer prevention and treatment. Further investigation is needed to validate the contribution of chrysin in tumour therapy in vivo.

\section{References}

1. Ren W, Qiao Z, Wang H, Zhu L and Zhang L: Flavonoids: promising anticancer agents. Med Res Rev 23: 519-534, 2003.

2. Ko HH, Tsao LT, Yu KL, Liu CT, Wang JP and Lin CN: Structure-activity relationship studies on chalcone derivatives. the potent inhibition of chemical mediators release. Bioorg Med Chem 11: 105-111, 2003.

3. Williams RJ, Spencer JPE and Rice-Evans C: Flavonoids: antioxidants or signalling molecules? Free Radic Biol Med 36: 838-849, 2004

4. Soobratte MA, Neergheen VS, Luximon-Ramma A, Aruoma OI and Bahorun T: Phenolics as potential antioxidant therapeutic agents: mechanism of action. Mutat Res 579: 200-213, 2005.

5. Cho H, Yun CW, Park WK, Kong JY, Kin KS, Park Y, Lee S and Kim BK: Modulation of the activity of pro-inflammatory enzymes, COX-2 and iNOS, by chrysin derivatives. Pharmacol Res 49: 37-43, 2004

6. Lapidot T, Walker MD and Kanner J: Antioxidant and prooxidant effects of phenolics on pancreatic beta-cells in vitro. $\mathrm{J}$ Agric Food Chem 50: 7220-7225, 2002.

7. Weng MS, Ho YS and Lin JK: Chrysin induces G1 phase cell cycle arrest in C6 glioma cells through inducing p21Waf1/Cip1 expression: involvement of p38 mitogenactivated protein kinase. Biochem Pharmacol 69: 1815-1827, 2005.

8. Pichichero E, Cicconi R, Mattei M, Gallinella-Muzi M and Canini A: Acacia honey and chrysin reduces proliferation of melanoma cells through alteration in cell cycle progression. Int J Oncol 37: 973-981, 2010.

9. Zhang T, Chen X, Qu L, Wu J, Cui R and Zhao Y: Chrysin and its phosphate ester inhibit cell proliferation and induce apoptosis in Hela cells. Bioorg Med Chem 12: 6097-6105, 2004.

10. Woo KJ, Jeong YJ, Park JW and Kwon TK: Chrysin induced apoptosis is mediated through caspase activation and Akt inactivation in U937 leukemia cells. Biochem Biophys Res Commun 325: 1215-1222, 2004.

11. Norbury CJ and Hickson ID: Cellular response to DNA damage. Annu Rev Pharmacol Toxicol 41: 367-401, 2001.

12. Hotchkiss RS, Strasser A, McDunn JE and Swanson PE: Mechanism disease - cell death. N Engl J Med 361: 1570-1583, 2009.

13. Galluzzi L, Maiuri MC, Vitale I, Zischka H, Castedo M, Zitvoge L and Kroemer G: Cell death modalities: classification and pathophysiological implications. Cell Death Differ 14: 1237-1266, 2007.

14. Elmore S: Apoptosis: a review of programmed cell death. Toxicol Pathol 35: 495-516, 2007.

15. Cory $\mathrm{S}$ and Adams JM: The $\mathrm{Bcl} 2$ family: regulators of the cellular life-or-death switch. Nat Rev Cancer 2: 647-656, 2002.

16. Kim H, Rafiuddin-Shah M, Tu HC, Jeffers JR, Zambetti GP Hsieh JJ and Cheng EHY: Hierarchical regulation of mitochondrion-dependent apoptosis by BCL-2 subfamilies. Nat Cell Biol 8: 1348-1358, 2006.

17. Fan TJ, Han LH, Cong RS and Liang J: Caspase family proteases and apoptosis. Acta Biochim Biophys Sin 37: 719-727, 2005.

18. Leszczyniecka M, Roberts T, Dent P, Grant S and Fisher PB: Differentiation therapy of human cancer: basic science and clinical applications. Pharmacol Ther 90: 105-156, 2001.

19. Horbinski $\mathrm{C}$ and Chu CT: Kinase signaling cascades in the mitochondrion: a matter of life or death. Free Radic Biol Med 38: 2-11, 2005.

20. Chuang SM, Wang IC and Yang JL: Roles of JNK, p38 and ERK mitogen-activated protein kinases in the growth inhibition and apoptosis induced by cadmium. Carcinogenesis 21: 1423-1432, 2000.

21. Bradford MM: A rapid and sensitive method for the quantitation of microgram quantities of protein utilizing the principle of protein-dye binding. Anal Biochem 72: 248-254, 1976.

22. Ickowicz Schwartz DI, Gozlan Y, Greenbaum L, Babushkina T, Katcoff DJ and Malik Z: Differentiation-dependent photodynamic therapy regulated by porphobilinogen deaminase in B16 melanoma. Br J Cancer 90: 1833-1841, 2004.
23. Hinnen P, de Rooij FWM, van Velthuysen MLF, Edixhoven A, van Hillegersberg R, Tilanus HW, Wilson JHP and Siersemal PD: Biochemical basis of 5-aminolaevulinic acid-induced protoporphyrin IX accumulation: a study patients with (pre) malignant lesion of oesophagus. Br J Cancer 78: 679-682, 1998.

24. Spencer JPE, Abd El Mhosen MM and Rice-Evans C: Cellular uptake and metabolism of flavonoids and their metabolites: implication for their bioactivity. Arch Biochem Biophys 423: 148-161, 2004

25. Depeint F, Gee JM, Williamson G and Johnson IT: Evidence for consistent patterns between flavonoid structures and cellular activities. Proc Nutr Soc 61: 97-103, 2002.

26. Mukai R, Shirai Y and Saito N: Subcellular localization of flavonol aglycone in hepatocytes visualized by confocal laser scanning fluorescence microscope. Cytotechnology 59: 177-182, 2009.

27. Walgren RA, Walle UK and Walle T: Transport of quercetin and its glucosides across human intestinal epithelial Caco-2 cells. Biochem Pharmacol 55: 1721-1727, 1998.

28. Zhanag S, Qin C and Safe SH: Flavonoids as aryl hydrocarbon receptor agonists/antagonists: effect of structure and cell context. Environ Health Perspect 11: 1877-1882, 2003.

29. Hodek P, Trefil P and Stiborova M: Flavonoids - potent and versatile biologically active compounds interacting with cytochromes P450. Chem Biol Interact 139: 1-21, 2002.

30. Alesiani D, Cicconi R, Mattei M, Montesano C, Bei R and Canini A: Cell cycle arrest and differentiation induction by 5,7dimethoxycoumarin in melanoma cell lines. Int J Oncol 32: 425-434, 2009.

31. Vena FCB, Turchiello RF, Laville I, Pigaglio S, Blais J and Tedesco AC: 5-aminolavulinic acid ester-induced protoporphirin IX in a murine melanoma cell line. Lasers Med Sci 19: 119-126, 2004.

32. Ortel B, Sharlin D, O'Donnell D, Sinha AK, Maytin EV and Hasan T: Differentiation enhances aminolevulinic acid-dependent photodinamic treatment of LNCaP prostate cancer cells. Br J Cancer 87: 1321-1327, 2002.

33. Alesiani D, Cicconi R, Mattei M, Bei R and Canini A: Inhibition of Mek 1/2 kinase activity and stimulation of melanogenesis by 5,7-dimethoxycoumarin. Int J Oncol 34: 1727-1735, 2008 .

34. Sato N, Moore BW, Keevey S, Drazba JA, Hasan T and Maytin EV: Vitamin D enhances ALA-induced protoporphyrin IX production and photodynamic cell death in 3-D organotypic cultures of keratinocytes. J Investig Dermatol 127: 925-934, 2007.

35. Siboni G, Amit-Patito I, Weizman E, Waintraub-Porat M, Weitman H, Ehrenberg B and Malik Z: Specificity of photosensitizer accumulation in undifferentiated versus differentiated colon carcinoma cells. Cancer Lett 196: 57-64, 2003.

36. Sinha AK, Anand S, Ortel BJ, Chang Y, Mai Z, Hasan T and Maytin EV: Methotrexate used in combination with aminolaevulinic acid for photodynamic killing of prostate cancer cells. Br J Cancer 95: 485-495, 2006.

37. Sassa S: Regulation of the genes for the heme pathway enzymes in erythroid and non-erythroid cells. Int J Cell Cloning 8: 10-26, 1990.

38. Greenbaum L, Katcoff DJ, Dou H, Gozlan Y and Malik Z: A porphobilinogen deaminase (PBGD) Ran-binding protein interaction is implicated in nuclear trafficking of PBGD in differentiating glioma cells. Oncogene 22: 5221-5228, 2003.

39. MacDonald IJ and Dougherty TJ: Basic principles of photodynamic therapy. J Porphyr Phthalocya 5: 105-129, 2001.

40. Pandey RK: Recent advances inphotodynamic therapy. J Porphyr Phthalocyanines 4: 368-373, 2000.

41. Namkoong J, Martino JJ and Chen S: From existing therapies to novel targets: a current view on melanoma. Front Biosci 11: 2081-2092, 2006.

42. Panka DJ, Atkins MB and Mier JW: Targeting the mitogenactivated protein kinase pathway in the treatment of malignant melanoma. Clin Cancer Res 12: 2371-2375, 2006.

43. Fueller J, Becker M, Sienerth AR, Fischer A, Hotz C and Galmiche A: C-RAF activation promotes BAD poly-ubiquitylation and turn-over by the proteasome. Biochem Biophys Res Commun 370: 552-556, 2008.

44. Enslen H, Brancho DM and Davis RJ: Molecular determinants that mediate selective activation of p38 MAP kinase isoforms. EMBO J 19: 1301-1311, 2000.

45. Zarubin $\mathrm{T}$ and Han $\mathrm{J}$ : Activation and signaling of the p38 MAP kinase pathway. Cell Res 15: 11-18, 2005. 
46. Hata K, Hori K and Takahashi S: Role of p38 MAPK in Lupeolinduced B16 2F2 mouse melanoma cell differentiation. J Biochem 134: 441-445, 2003.

47. Ivanov VN and Ronai Z: p38 protects human melanoma cells from UV induced apoptosis through down-regulation of NFkappaB activity and Fas expression. Oncogene 19: 3003-3012, 2000.

48. Edlund S, Bu S, Schuster N, Aspenström P, Heuchel R, Heldin NE, Ten Dijke P, Heldin CH and Landstrom M: Transforming growth factor-betal (TGF-beta)-induced apoptosis of prostate cancer cells involves Smad7-dependent activation of p38 by TGF-beta-activated kinase 1 and mitogen-activated protein kinase kinase 3. Mol Biol Cell 14: 529-544, 2003.

49. Van Leathem A, Van Kelst S, Lippens S, Declercq W, Vandenabeele P, Janssens S, Vandenheede JR, Garmyn M and Agostinis P: Activation of p38 MAPK is required for Bax translocation to mitochondria, cytochrome c release and apoptosis induced by UVB irradiation in human keratinocytes. FASEB J 18: 1946-1948, 2004.
50. Sheng G, Guo J and Warner BW: Epidermal growth factor receptor signaling modulates apoptosis via p38alpha MAPKdependent activation of Bax in intestinal epithelial cells. Am J Physiol Gastrointest Liver Physiol 293: G599-G606, 2007.

51. Jang BC, Lim KJ, Paik JH, Cho JW, Baek WK, Suh MH, Park JB, Kwon TK, Park JW, Kim SP, Shin DH, Song D, Bae JH, Mun KC and Suh SI: Tetrandrine-induced apoptosis is mediated by activation of caspases and PKC-d in U937 cells. Biochem Pharmacol 67: 1819-1829, 2004.

52. Gross A, McDonnell JM and Korsmeyer SJ: Bcl-2 family members and the mitochondrial in apoptosis. Genes Dev 13: 1899-1911, 1999.

53. Porter AG and Janicke RU: Emerging roles of caspase-3 in apoptosis. Cell Death Differ 6: 99-104, 1999. 